Review

\title{
The choroid plexus in health and in disease: dialogues into and out of the brain
}

\author{
Fernanda Marques ${ }^{\mathrm{a}, \mathrm{b}}$, João Carlos Sousa ${ }^{\mathrm{a}, \mathrm{b}}$, Maria Alexandra Brito ${ }^{\mathrm{c}}$, Jens Pahnke ${ }^{\mathrm{d}, \mathrm{e}, \mathrm{f}}$, Cecilia Santos ${ }^{\mathrm{g}}$, \\ Margarida Correia-Neves ${ }^{\mathrm{a}, \mathrm{b}}$, Joana Almeida Palha ${ }^{\mathrm{a}, \mathrm{b}, *}$ \\ a Life and Health Sciences Research Institute (ICVS), School of Health Sciences, University of Minho, Braga, Portugal \\ b ICVS/3B's-PT Government Associate Laboratory, Braga/Guimarães, Portugal

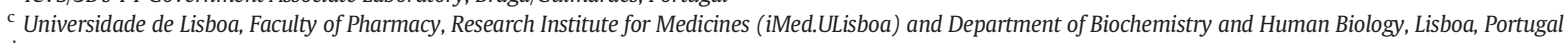 \\ ${ }^{d}$ University of Oslo (UiO) and Oslo University Hospital (OUS), Department of Neuro-/Pathology, Translational Neurodegeneration Research and Neuropathology Lab, Oslo, Norway \\ e University of Lübeck, LIED, Lübeck, Germany \\ ${ }^{\mathrm{f}}$ Leibniz Institute for Plant Biochemistry, Halle, Germany \\ ${ }^{g}$ CICS-UBI - Health Sciences Research Centre, University of Beira Interior, Av. Infante D. Henrique, 6200-506 Covilhã, Portugal
}

\section{A R T I C L E I N F O}

\section{Article history:}

Received 25 May 2016

Revised 20 July 2016

Accepted 17 August 2016

Available online 18 August 2016

\section{Keywords:}

Choroid plexus

Cerebrospinal fluid

Transporters

Neonatal jaundice

Multiple sclerosis

Alzheimer's disease

Gender

Aging

Neurogenesis

\begin{abstract}
A B S T R A C T
This article brings the choroid plexus into the context of health and disease. It is remarkable that the choroid plexus, composed by a monolayer of epithelial cells that lie in a highly vascularized stroma, floating within the brain ventricles, gets so little attention in major physiology and medicine text books and in the scientific literature in general. Consider that it is responsible for producing most of the about $150 \mathrm{~mL}$ of cerebrospinal fluid that fills the brain ventricles and the subarachnoid space and surrounds the spinal cord in the adult human central nervous system, which is renewed approximately 2-3 times daily. As such, its activity influences brain metabolism and function, which will be addressed. Reflect that it contains an impressive number of receptors and transporters, both in the apical and basolateral sides of the epithelial cells, and as such is a key structure for the communication between the brain and the periphery. This will be highlighted in the context of neonatal jaundice, multiple sclerosis and Alzheimer's disease. Realize that the capillaries that irrigate the choroid plexus stroma do not possess tight junctions and that the blood flow to the choroid plexus is five times higher than that in the brain parenchyma, allowing for a rapid sensing system and delivery of molecules such as nutrients and metals as will be revised. Recognize that certain drugs reach the brain parenchyma solely through the choroid plexus epithelia, which has potential to be manipulated in diseases such as neonatal jaundice and Alzheimer's disease as will be discussed. Without further notice, it must be now clear that understanding the choroid plexus is necessary for comprehending the brain and how the brain is modulated and modulates all other systems, in health and in disease. This review article intends to address current knowledge on the choroid plexus, and to motivate the scientific community to consider it when studying normal brain physiology and diseases of the central nervous system. It will guide the reader through several aspects of the choroid plexus in normal physiology, in diseases characteristic of various periods of life (newborns-kernicterus, young adults-multiple sclerosis and the elderAlzheimer's disease), and how sex-differences may relate to disease susceptibility.
\end{abstract}

(c) 2016 Elsevier Inc. All rights reserved.

\section{Contents}

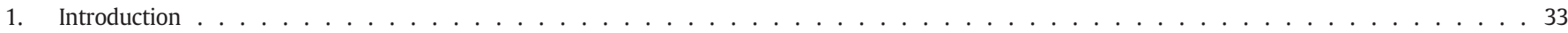

2. Essential features of the cellular/tissue anatomy and function . . . . . . . . . . . . . . . . . . . . . . . . . . . . . . .

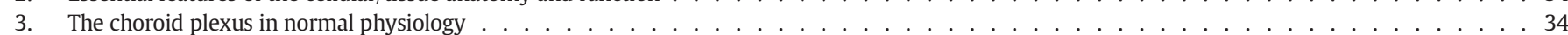

Abbreviations: $A D$, Alzheimer's disease; $A B$, amyloid beta; $A B C$, ATP-binding cassette; P-gp, ABCB1, ABC transporters P-glycoprotein; $B B B$, blood-brain barrier; $B C S F B$, blood-CSF

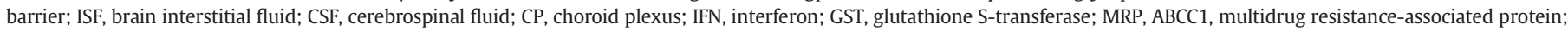
MS, multiple sclerosis; UCB, unconjugated bilirubin; UGT, uridine diphosphoglucuronosyl transferase.

* Corresponding author at: Life and Health Sciences Research Institute (ICVS), School of Health Sciences, University of Minho, Campus Gualtar, $4710-057$ Braga, Portugal.

E-mail addresses: fmarques@ecsaude.uminho.pt (F. Marques), jcsousa@ecsaude.uminho.pt (J.C. Sousa), abrito@ff.ulisboa.pt (M.A. Brito), jens.pahnke@medisin.uio.no (J. Pahnke), csantos@fcsaude.ubi.pt (C. Santos), mcorreianeves@ecsaude.uminho.pt (M. Correia-Neves), japalha@ecsaude.uminho.pt (J.A. Palha).

Available online on ScienceDirect (www.sciencedirect.com). 
3.1. Sex differences. . . . . . . . . . . . . . . . . . . . . . . . . . . . . . . . . . . . . 34

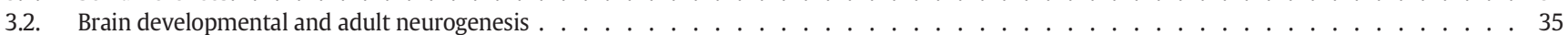

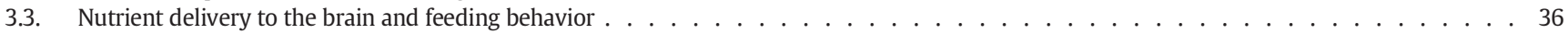

3.4. Metal homeostasis . . . . . . . . . . . . . . . . . . . . . . . . . . . . . 36

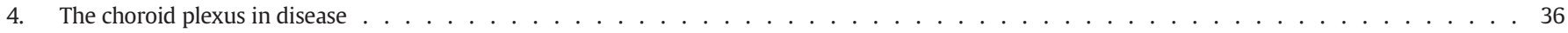

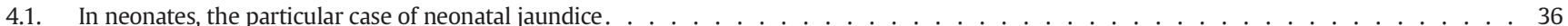

4.2. In young adults, in multiple sclerosis. . . . . . . . . . . . . . . . . . . . . . . . .

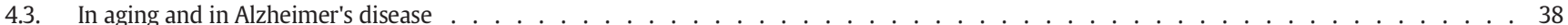

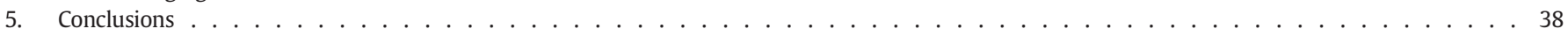

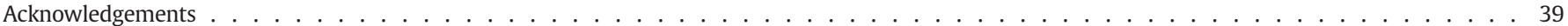

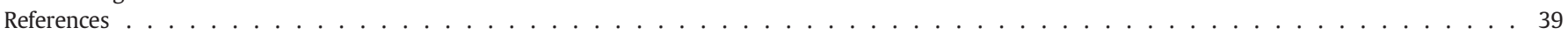

\section{Introduction}

The brain parenchyma is protected from the large variations in the concentration of the various molecules that circulate in the blood in diverse physiological and pathological circumstances. After a protein rich meal, for instance, the brain is protected from the subsequent increase in the blood glutamate concentration, which would otherwise result in a hyper excitability state since glutamate is an excitatory neurotransmitter. Similarly, the brain does not uptake the same concentration of several pharmaceutical compounds administered to successfully treat diseases of the periphery. While these passage restrictions are beneficial, they may otherwise become undesirable when considering the need to have drugs that reach the brain to treat diseases such as brain tumors or dementia. Of notice, similar reasoning must be envisaged for molecules of brain metabolism that need to be eliminated. In Alzheimer's disease (AD), the increase in the formation of the amyloid beta $(A B)$ peptide that ultimately deposits to form amyloid plaques may theoretically be prevented if mechanisms to increase its elimination out of the brain become successful.

The complexity of getting into and out of the brain resides on the brain barriers. Understanding their function and relevance goes beyond their strict role as gates for the brain; the barriers themselves display specific mechanisms and metabolic pathways that define the composition of the cerebrospinal fluid (CSF) and the brain interstitial fluid (ISF)

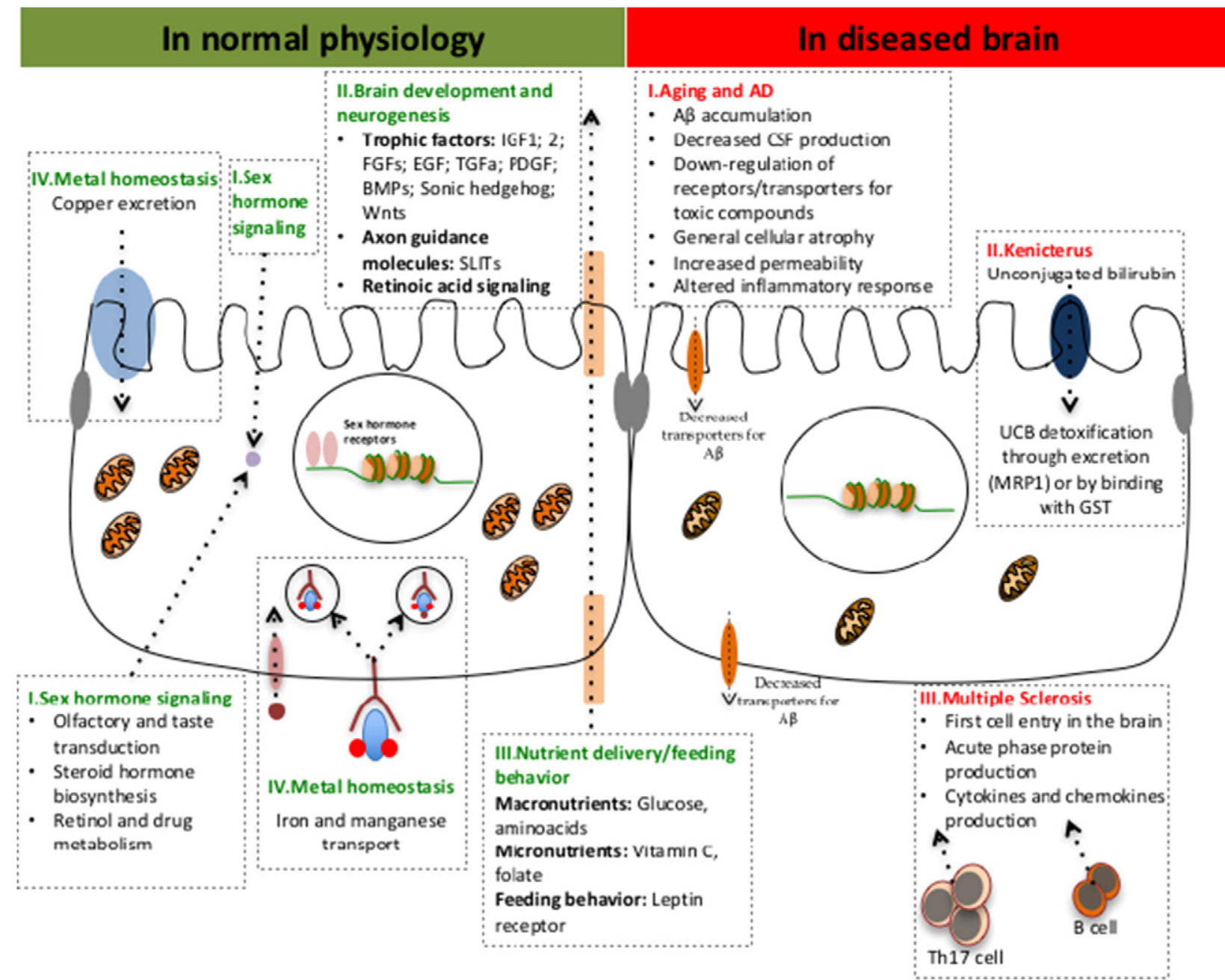

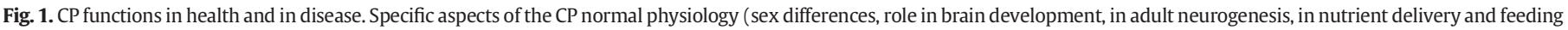

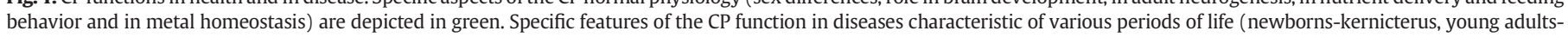
multiple sclerosis and the elder-AD) are depicted in red. 
in health and in disease. While the blood-brain barrier (BBB), formed by the endothelial cells of the capillaries that irrigate the brain parenchy$\mathrm{ma}$, is the best well studied and known brain barrier, this review will focus on the blood-CSF barrier (BCSFB) formed by the choroid plexus (CP) epithelial cells. Starting with a general overview of the CP anatomy and better described functions and challenges, this review then follows to address particular aspects of the normal physiology (sex differences, role in brain development and in adult neurogenesis and in nutrient delivery and feeding behavior), in metal homeostasis and, finally, specific features of the $\mathrm{CP}$ function in diseases characteristic of various periods of life (newborns-kernicterus, young adults-multiple sclerosis (MS) and the elder-AD). Fig. 1 depicts the overall message of this review.

\section{Essential features of the cellular/tissue anatomy and function}

The $\mathrm{CP}$ is a tissue that floats in the brain ventricles, composed of a monolayer of epithelial cells that lie in a highly vascularized stroma of connective tissue rich in fibroblasts and cells of the immune system. The monolayer of CP epithelial cells originates from the ependymal cells that circumvent the brain ventricles. The epithelial cells of the $\mathrm{CP}$ are bound by tight junctions (Wolburg and Paulus, 2010), which confers them the barrier properties to prevent paracellular diffusion of substances. As such, the monolayer of epithelial cells is what constitutes the BCSFB, since the capillaries in the $\mathrm{CP}$ stroma are fenestrated (there is no BBB in the capillaries that irrigate the $\mathrm{CP}$ stroma). Another interesting feature of the $\mathrm{CP}$ vasculature is that the blood flow rate at the $\mathrm{CP}$ is about five times higher than that of other brain regions (Maktabi et al., 1990).

Anatomically, the $\mathrm{CP}$ is a small structure when compared to the brain volume, easily observed when dissecting the brain. However, given the structural characteristics of the epithelial cells, which contain microvilli, the total surface area is estimated to be from about 25 to $50 \%$ of the total surface area of the capillaries that irrigate the brain $\left(2-5 \mathrm{~m}^{2}\right.$ versus $10 \mathrm{~m}^{2}$ ) (Spector et al., 2015), even though this is still a controversial issue (Damkier et al., 2013). These anatomical and structural features are of relevance for the $\mathrm{CP}$ secretory and transport functions. In accordance, the $\mathrm{CP}$ epithelial cells are enriched in mitochondria, which are also essential to provide the energy required for active transport and secretion.

The CP main and better described function is the production of CSF. The CSF fills the brain ventricles and the subarachnoid space that surrounds the central nervous system. The adult human has about $150 \mathrm{~mL}$ of CSF which is renewed 2-3 times per day (Cserr, 1971). This volume is impressive and in itself highlights the importance of the $\mathrm{CP}$. The ventricular CSF exchanges with the ISF of the brain parenchyma since the ependymal cells that surround the ventricles do not constitute a barrier. The CSF from the subarachnoid space also exchanges with the brain ISF through periarterial influx at the Virchow-Robin spaces (Iliff et al., 2013), as next discussed further.

The best described pathway for CSF excretion to the peripheral circulation occurs mostly through the arachnoid granulations and arachnoid villi (Chen et al., 2015). However, there is evidence supporting that the CSF also drains into extracranial lymphatic vessels and cervical lymph nodes (Aspelund et al., 2015; Chen et al., 2015; Louveau et al., 2015). The CSF pathway into the lymph nodes is now better understood given the finding of a lymphatic vessel network in the dura mater of the mouse brain. It was shown that dural lymphatic vessels absorb CSF from the adjacent subarachnoid space and brain ISF via the glymphatic system (Aspelund et al., 2015). The glymphatic system is a recently described system important for the clearance of the waste products from the brain: the CSF from the subarachnoid space is driven into the Virchow-Robin spaces where it passes through the periarterial influx to the brain parenchyma. Once in the parenchyma, CSF exchanges with the ISF facilitating the clearance of solutes and waste products from the brain (Iliff et al., 2013). This process is known to be dependent on the expression of aquaporin 4 , expressed by astrocytes, since animals lacking this water channel exhibit slowed CSF influx through this system and a $~ 70 \%$ reduction in ISF solute clearance (Iliff et al., 2012).
This exchange of solutes between the CSF and the ISF is driven by arterial pulsation and regulated during sleep (when the glymphatic activity is dramatically enhanced, while its function is suppressed during wakefulness) (Mendelsohn and Larrick, 2013). Altogether, how the CSF and the brain ISF contents ultimately reach the periphery represents one of the challenges to be addressed and that may contribute to the greater puzzle of getting into and out of the brain and will certainly be a hot topic of research in health and in disease.

\section{The choroid plexus in normal physiology}

\subsection{Sex differences}

Little is known about sex-specificities of the $\mathrm{CP}$ and how this may contribute to the observed sex-dependent prevalence differences in some diseases of the central nervous system (CNS). There is however mounting evidence that the $\mathrm{CP}$ is highly sensitive to sex hormones and differently regulated between sexes. The $\mathrm{CP}$ expresses receptors for the sex hormones (i.e. progesterone, oestrogens alpha and beta and androgen). Once bound by the cognate hormones these receptors modulate the expression of many genes and influence the composition of the CSF (Alves et al., 2009; Hong-Goka and Chang, 2004; Quadros et al., 2007; Quintela et al., 2013; Quintela et al., 2016). Hormone withdrawal or reduction upon gonadectomy has profound impact on the $\mathrm{CP}$ transcriptome. Whole transcriptome studies showed that ovariectomised female rats display about $10 \%$ of the genes with at least 1.5 fold difference in expression (1168 up- and 1328 down-regulated) when compared to sham-operated females. Interestingly, gonadectomy results solely in a differential expression of $2.2 \%$ of the transcriptome (426 up- and 123 down-regulated genes). Among the pathways regulated by the hormonal background are those involved in olfactory and taste transduction, which are functional in the $\mathrm{CP}$ and may constitute important means to survey the chemical composition of the CSF and blood (Goncalves et al., 2016; Tomas et al., 2016). Also altered are pathways involved in steroid hormone biosynthesis, retinol metabolism and drug metabolism (Quintela et al., 2013) (Fig. 1). The major differences in the $\mathrm{CP}$ transcriptome of sham male and female rats, as identified by cDNA microarrays studies, are related with circadian rhythm signaling, and with several canonical pathways associated with metabolism, the barrier function of the $\mathrm{CP}$ and stem cell differentiation (Quintela et al., 2016) (Table 1). The establishment of circadian

\section{Table 1}

Choroid plexus genes whose expression is sex-dependent in specific pathways of relevance for selected physiological and disease processes.

\begin{tabular}{|c|c|c|}
\hline Pathways & Genes & Fold change \\
\hline \multirow[t]{5}{*}{ Circadian rhythm signaling } & Arntl & -4.30 \\
\hline & Per2 & 3.59 \\
\hline & $D b p$ & 3.58 \\
\hline & Ciart & 8.37 \\
\hline & Per3 & 2.00 \\
\hline \multirow[t]{15}{*}{ Neurogenesis and stem cell differentiation } & Sfrp2 & -59.77 \\
\hline & Ноха2 & -35.70 \\
\hline & HoxA5 & -35.70 \\
\hline & Gfra1 & -11.05 \\
\hline & Hoxb5 & -8.15 \\
\hline & Gata4 & -3.02 \\
\hline & $R b p 1$ & -3.05 \\
\hline & $S f n$ & -3.89 \\
\hline & Inadl & -2.56 \\
\hline & Ppm1j & -2.15 \\
\hline & $\lg f 1$ & -1.98 \\
\hline & $\operatorname{Tgfa}$ & -1.59 \\
\hline & $\lg f 2 b p 1$ & 3.84 \\
\hline & $R d h 5$ & 2.00 \\
\hline & $\lg 2$ & 1.03 \\
\hline \multirow[t]{2}{*}{ Immune cell trafficking } & Ccr6 6 & 1283 \\
\hline & Ccl20 & -1.66 \\
\hline
\end{tabular}

-: fold down-regulated; + : fold up-regulated. Fold changes in males. 
rhythms influences several aspects of human physiology from the sleep/ vigil cycles to the fine tune of cell function. Drug metabolism, distribution and absorption are known to oscillate in a circadian pattern thereby affecting pharmacokinetics (Baraldo, 2008). Immune system responses to infectious agents and inflammation are also set by the circadian recruitment of immune cells to tissues and daily oscillations in the expression of pro-migratory factors by immune cells (Scheiermann et al., 2013). As a circadian oscillator, with differences in the clock machinery between males and females, the CP may well establish the circadian pace for drug metabolism and absorption and for the immune responses of the CNS. In this respect, transcriptional differences possibly account for the sex-related differences in the response to pharmacological treatments like anesthesia and antidepressants (Mawhinney et al., 2013; Bigos et al., 2009), or in the immune function (Butts and Sternberg, 2008).

Some examples of genes up-regulated in males are those associated with the establishment of circadian rhythms, such as Ciart (circadian associated repressor of transcription), a recently identified key element of the circadian oscillator that represses the transcriptional activity of the CLOCK (clock circadian regulator)-ARNTL(aryl hydrocarbon receptor nuclear translocator like) heterodimer (Mitsui et al., 2001). Alike, Per (period circadian clock) 2 and Per 3 are up-regulated in the CP of males in comparison to females. $D b p$ (D-box binding PAR bZIP transcription factor), or albumin promoter (D site), is also a transcriptional activator that modulates important clock output genes, is also up-regulated in the male $\mathrm{CP}$ compared to females' (Belmadani et al., 2006). Arnt, on the other hand, is downregulated in males (Quintela et al., 2016) (Table 1).

Interestingly, age-related alterations in the expression of circadian genes in the CP have been described in mice (Mesquita et al., 2015), and these may contribute to alterations in the barrier properties with age (He et al., 2014).

Given the role of $\mathrm{CP}$ secreted molecules in the neurogenesis of the subventricular zone (Falcao et al., 2012a), it is also important to note that genes involved in embryonic stem cell differentiation, retinoic acid biosynthesis and Hippo signaling, all of them processes associated with neurogenesis, are down-regulated in the $\mathrm{CP}$ of males compared to females (2-fold or above) (Table 1). These include Gata4 (GATA binding protein 4) and Hoxb5 (homeobox B5) that regulate human embryonic stem cell renewal and differentiation (Fessner et al., 2014) and vascular remodeling (Okada et al., 2004). Genes associated with the visual cycle/retinoate biosynthesis [Rbp1 (retinol binding protein 1) and Rdh5 (retinol dehydrogenase 5)], which are seminal elements of the retinoic acid metabolism, also fundamental for stem cell differentiation and frequently used to promote neural differentiation of pluripotent cells into neural cells (Mo et al., 2014), are also down-regulated in males in comparison to females (Quintela et al., 2016). In addition, the Hippo signaling pathway that plays a key role in tissue homeostasis and organ size control by regulating tissue-specific stem cells (Inestrosa and Varela-Nallar, 2014) is also down-regulated in males. Moreover, among the 10 most differently expressed genes between males and females several are related to neurogenesis, including Sfrp2 (secreted frizzled related protein 2), a modulator of the Wnt signaling pathway (Combarros et al., 2010); Gfra1 (GDNF family receptor alpha 1 ), a potent neurotrophic factor involved in the control of neuron survival and differentiation (Falcao et al., 2012a) and C Cr1 (C-C motif chemokine receptor 1 ), a $\mathrm{G}$ protein coupled receptor that has also been associated with stem cell proliferation (Bell et al., 2013). Moreover, the Igf2bp1 (insulin-like growth factor 2 mRNA binding protein 1), which controls cell polarization, migration, morphology, metabolism, proliferation and differentiation during embryogenesis, and is required for proper nerve cell migration and morphological development is nearly 4 fold up-regulated in males in comparison to females (Strazielle and Ghersi-Egea, 2015). These data further support that the synthesis of peptides implicated in neurogenesis and neuronal survival have seminal functions in the $\mathrm{CP}$ that remarkably differ between males and females.
Therefore, different hormonal levels, as seen between males and females and sex hormone decline throughout aging are likely to regulate $\mathrm{CP}$ gene and protein expressions and function. Their relevance in the context of age-related differences in diseases of the CNS such as the propensity for depression or the increased prevalence of MS in women (Dunn et al., 2015) deserves further studies.

\subsection{Brain developmental and adult neurogenesis}

The CP-CSF system is much studied under conditions of altered brain function or brain malfunction, namely in neurodegenerative disorders such as MS and AD, but currently further attention is given to its role in nurturing the brain with essential molecules that regulate normal brain function. In addition, some recent studies have pinpointed the critical role of $\mathrm{CP}$ secreted molecules in the regulation of neural stem cells, highlighting the essential contribution of the CP-CSF for CNS maturation and function. The importance of the $\mathrm{CP}-\mathrm{CSF}$, and $\mathrm{CP}$-borne molecules, and how CP malfunction during development dramatically influences brain formation was extensively reviewed previously (Lehtinen and Walsh, 2011; Lun et al., 2015b). One of such molecules, secreted from the CP into the CSF, is insulin-growth factor 2 , shown to promote growth and neuronal survival in the mouse developing cortex (Lehtinen et al., 2011). One further example is retinoic acid signaling (Parada et al., 2008), essential for cortical neuron formation and migration to the cerebral cortex (Crandall et al., 2011). The currently available data, namely from embryonic $\mathrm{CP}$ transcriptome studies both in rodents (Saunders et al., 2015) and non-human primates (Ek et al., 2015), suggest that the $\mathrm{CP}$ must display a temporal highly heterogeneous secretome during development; furthermore, region specific (lateral vs 4th ventricle $\mathrm{CP}$ ) gene expression patterns confer a positional hallmark to CP function during development (Lun et al., 2015a; Strazielle and Ghersi-Egea, 2015). Given these temporal expression patterns during embryonic and early brain postnatal development, it is reasonable to anticipate that the molecules secreted by the $\mathrm{CP}$ might also regulate embryonic and early adulthood brain cells function, a topic to be explored.

Considering how CP secreted factors influence neural stem cells, it is of interest that the adult brain displays 2 major sites of neurogenesis, the subventricular zone, in close proximity with the $\mathrm{CP}$ and directly bathed by the CSF; and, the subgranular zone of the dentate gyrus of the hippocampus, also under the influence of the CSF content. The capacity of the brain to generate new neurons is of relevance both physiologically and in disease contexts. Given the CP role in CSF synthesis and the type of proteins it secretes, namely trophic factors, and the close contact between the CSF and neural stem cells of the neurogenic niches, it is expected that the $\mathrm{CP}$ plays a role in the regulation of adult neurogenesis. Of interest, both adult niches display topographical specificities in the intrinsic phenotype and properties of the neural stem cells (Falcao et al., 2012b; Fiorelli et al., 2015), which further adds to the complex interaction that might arise between CP-CSF derived molecules and the adult brain stem cell niches. Comprehensive analysis of the adult CP transcriptome and secretome, both under physiological conditions (Marques et al., 2011; Thouvenot et al., 2006) and in disease (Marques et al., 2012; Marques et al., 2009b; Mesquita et al., 2015), unraveled the expression of several of these genes encoding key molecules known to modulate neural stem cells of either the subventricular zone or the dentate gyrus (or both). Among these are insulin-like growth factors 1 and 2, several members of the fibroblast growth factor family, epidermal growth factor, transforming growth factor alpha, platelet derived growth factors, bone morphogenetic proteins, sonic hedgehog, Wnts and axon guidance molecules such as Slits (Falcao et al., 2012a) (Fig. 1). Thus, understanding the exact contribution of each of these $\mathrm{CP}$ derived trophic factors to the modulation of the neurogenic niches and other cells of the brain parenchyma is of relevance. This may provide clues for the treatment of neurological disorders such as depression, $\mathrm{AD}$ and $\mathrm{MS}$, and again may unravel sex-differences in disease susceptibility. 


\subsection{Nutrient delivery to the brain and feeding behavior}

Similar to the BBB endothelial cells, the CP epithelial cells are endowed with a plethora of specific carriers that convey to the CSF, and hence to the brain parenchyma, a range of nutrients that are critical to support brain function. Among these are major nutrients such as glucose and amino acids, but also essential micronutrients such as ascorbate (vitamin C) and folate (Redzic and Segal, 2004; Spector et al., 2015). The transport of ascorbate and folate via the $\mathrm{CP}$ to the brain is of particular relevance given that: i) ascorbate is not synthetized in the brain and its transporter is highly expressed in the $\mathrm{CP}$, but absent from the $\mathrm{BBB}$; ii) both molecules are 4 times more concentrated in the CSF than in the blood; iii) knockout mice for both ascorbate and folate CP transporters display impaired development and fail to thrive while displaying undetectable levels of these micronutrients in the brain; and, iv) the absolute requirement of these nutrients for the neuronal metabolism of the deoxyribonucleoside 5-hydroxymethyldeoxycytidine, and specifically of ascorbic acid as an essential cofactor of Tet (ten-eleven translocation) enzymes that participate in DNA demethylation in mammals (Keep and Smith, 2011; Spector and Johanson, 2014; Yin et al., 2013).

One other relevant role for the $\mathrm{CP}$ concerning nutrients homeostasis relates with the regulation of feeding behavior by several peripheral factors, among which leptin (the adipocyte-derived obesity gene product) has been thoroughly studied (Murray et al., 2014). Produced mostly by adipose tissue, leptin interacts with receptors in the $\mathrm{CP}$ and in the median eminence (a circumventricular organ of the brain where endothelial cells are deprived of barrier proprieties), which internalize it into the CSF (Dietrich et al., 2008; Mitchell et al., 2009). Leptin is a key hormone peptide for the regulation of energy balance that has several CNS actions, namely by acting at leptin receptors in the arcuate nucleus of the hypothalamus and hence regulating feeding behavior. Exactly how blood circulating leptin readily ( $\sim 20 \mathrm{~min}$ after a peripheral i.v. injection of radiolabeled ${ }^{125}$ I-leptin) distributes to the arcuate nucleus (Banks et al., 1996) is still not totally clear. While in vitro experiments suggest that a receptor mediated transporter system exists at the BBB (Golden et al., 1997), the in vivo experiments with ${ }^{125}$ I-leptin indicate that uptake of peripheral leptin occurs via the $\mathrm{CP}$ and the median eminence. From these 2 structures, leptin rapidly reaches the CSF and can be taken to the arcuate nucleus that is in direct contact with the brain ventricles filled with CSF (Smith and Ferguson, 2014). Interestingly, leptin receptors are also highly expressed in the $\mathrm{CP}$ and are regulated by both leptin levels and nutritional status (Mitchell et al., 2009) (Fig. 1); nevertheless, the relevance of existing leptin receptors in the $\mathrm{CP}$ is not understood and so the role of the $\mathrm{CP}$ in leptin transport might be limited to readily supplying a peripheral signal (leptin) to the brain parenchyma, or it may otherwise relate with a more general role in the regulation of feeding behavior.

\subsection{Metal homeostasis}

Metals such as iron, copper and manganese are essential elements mostly as co-factors of several enzymes. Given their high oxidative properties, their free concentration must be tightly regulated to prevent damage of molecular and cellular structures. Understanding metal homeostasis requires knowledge not only on the need for absorption from the external milieu in the diet, to transport in the circulation, uptake by tissues, incorporation into the proteins for function and/or storage, and excretion. In addition to metals with known described physiological functions, increased concern relates with exposure to metals derived from environmental pollution such as the case for lead. Dysregulation of metal availability for the brain has been associated with brain disorders including AD, Parkinson's disease or fragile X-associated tremor/ataxia syndrome (Ariza et al., 2015; Bakulski et al., 2012; Zheng and Monnot, 2012). The brain barriers are of relevance as the interface between the periphery and the brain to regulate metal homeostasis, which will be briefly exemplified for the $\mathrm{CP}$ with respect to copper, manganese, iron and lead.

Copper is required for the activity of enzymes such as cytochromes and ceruloplasmin; its entry into the brain mostly occurs at the $\mathrm{BBB}$, while the $\mathrm{CP}$ has been described as the main route for excretion from the CSF into the blood (Fu et al., 2014). Copper toxicity induced by manganese seems to be mediated by the decrease in the excretion route and the consequent accumulation of copper in the CSF (Fu et al., 2014).

Manganese is a cofactor of key enzymes involved in the scavenging of free radicals (Bresciani et al., 2015). Manganese is transported into the brain parenchyma both by the BBB and the BCSFB; when in excess, it is mostly transported across the CP into the CSF. Interestingly, manganese can be transported either by metal transporters and channels or by transcytosis with the transferrin receptor (Fitsanakis et al., 2007; Yokel, 2009); which brings together the transport of manganese and iron.

Iron transfer into the brain occurs mostly by transcytosis of transferrin upon binding to its cognate receptors, which occurs both at the BBB and BCSFB. Of notice, the role of the $\mathrm{CP}$ seems to be crucial for iron brain homeostasis, given the unique presence of a set of proteins known to finely determine iron absorption from the intestine lumen and delivery to the circulation by the enterocyte (Rouault et al., 2009). Hepcidin is the liver regulatory hormone secreted when there is sufficiency of iron; hepcidin prevents iron release into the circulation from the enterocyte through internalization of ferroportin. Since there is no mechanism for the body to excrete iron, except through bleeding or physiological intestinal cell renewal, iron homeostasis must be tightly regulated. Of notice, the $\mathrm{CP}$ has also been shown to produce hepcidin in specific conditions such as in response to peripheral inflammation (Marques et al., 2009a). The observation that the CP can have an endocrine role mimicking that of the liver, in this case regulating iron availability for the brain (Marques et al., 2009a), is of great interest when considering that this may restrict iron availability for microorganism entering the brain, on one side, or iron accumulation that is observed in several diseases of the CNS such as AD (Mesquita et al., 2012) and Parkinson's disease (Jiang et al., 2016), on the other.

Lead is a recognized neurotoxic pollutant known for long to accumulate in the CP (Zheng, 2001). Several studies provided evidence that this accumulation mediates some of its neurotoxic effects, such as increasing the accumulation of the Alzheimer's $A \beta$ peptide through decrease of the of low density lipoprotein receptor protein 1 that participates in $A \beta$ clearance out of the brain (Behl et al., 2010).

The $\mathrm{CP}$ is also the site of synthesis of metallothioneins (MT) $1 / 2$ and 3 (Goncalves et al., 2008; Martinho et al., 2010). Under physiological conditions, MT-1/2s and MT-3 bind zinc and copper through their cysteine residues, but these metals can be replaced by others such as cadmium, mercury, silver, platinum and lead (Faller, 2010). The high MTs metal binding capacity confers them an essential role in metal cellular homeostasis and heavy metal detoxification, protecting cells and tissues against their toxicity (Maret, 2009).

Altogether, when considering metal homeostasis and metal-induced toxicity, it becomes clear that the $\mathrm{CP}$ is of relevance not only given the presence of transporters, channels and receptors, but also as a site of hormonal regulation of the transport mechanisms mediating metal delivery into and/or excretion out of the brain, both in normal brain physiology and when associated with neurotoxicity and brain damage.

\section{The choroid plexus in disease}

\subsection{In neonates, the particular case of neonatal jaundice}

Few laboratories have addressed the ontogeny of the CP function, and the misconception of immaturity of the brain barriers during development remains, despite the evidence on the contrary (Liddelow et al., 2013; Saunders et al., 2015). It is however now clear that the ontogeny of the $\mathrm{CP}$ transcriptome will provide further evidence on its role in 
health and disease throughout life (Kratzer et al., 2013; Liddelow et al., 2013; Marques et al., 2009a; Marques et al., 2009b; Marques et al., 2011; Mesquita et al., 2015; Saunders et al., 2015). Neonatal jaundice exemplifies the contribution of the $\mathrm{CP}$ in a disease that affects newborns.

In neonates, serum unconjugated bilirubin (UCB) is usually elevated in the first 2 weeks of postnatal life due to the features of bilirubin metabolism in perinatal life, namely the increased breakdown of fetal erythrocytes, the deficient albumin transport to the liver, and decreased conjugation, leading to a condition known as physiological jaundice of the newborn, which is very frequent, usually benign and requiring no treatment (Brito et al., 2006). However, in some newborn infants, plasma UCB levels can increase dramatically owing to impaired postnatal maturation of hepatic transport or conjugation of UCB, or augmented hemolysis. This jaundice is pathologic and may lead to death or to severe neurodevelopmental sequelae in survivors, a devastating condition known as kernicterus (Brito et al., 2006). Despite the knowledge about the neurotoxic potential of UCB (Brites and Brito, 2012), there are still no reliable indicators of patients at risk of bilirubin-induced neurologic dysfunction. As so, kernicterus cases have been reported, namely in premature infants presenting total bilirubin levels below those referenced for clinical intervention (Ahdab-Barmada and Moossy, 1984; Merhar and Gilbert, 2005). These facts indicate that hyperbilirubinemia in newborns remains an area requiring attention and evaluation.

UCB can enter brain parenchyma either directly via the BBB, or by specific uptake across the CP (Ghersi-Egea et al., 2009) (Fig. 1). Free UCB may leave the brain by diffusion through the abluminal membrane of the endothelial cells or following drainage into the CSF across the apical membrane of the CP epithelial cells (Ghersi-Egea et al., 2009). Alternatively, the clearance of UCB from the CNS may be mediated by transporter(s) expressed in brain capillary endothelium and in CP epithelial cells, which mediate the active export of UCB back into plasma, thus limiting the concentrations of unbound UCB (known as free UCB) in the CSF and brain parenchyma (Ghersi-Egea et al., 2009; Ostrow et al., 2004). Clearance of UCB by the CSF is of particular interest during the neonatal period, as it provides a means to compensate for its entrance into the brain, helping to keep UCB load in the brain low.

The $\mathrm{CP}$ metabolism also here shows resemblance with that of the liver, as seen in the case of iron homeostasis. In accordance, now in the context of bilirubin, the uridine diphosphoglucuronosyl transferase (UGT) activity of rat CP epithelial cells is comparable to that of the liver (Ghersi-Egea et al., 1994). This suggests that the choroidal tissue may constitute an important site for UCB detoxification in the brain, inasmuch the $\mathrm{CP}$ epithelial cells are equipped with a number of transporters, particularly of the multidrug resistance-associated protein (MRP; ABCC1) family, that might account for the secretion of bilirubin conjugates towards the blood (Ghersi-Egea et al., 2009), similarly to what happens in the liver towards the bile. However, no reports are available on the expression of the bilirubin conjugating enzyme throughout development, and therefore its contribution in neonatal jaundice remains unclear. Also in line with the "liver-like" function, the CP express glutathione S-transferase (GST) proteins (Gazzin et al., 2012a), which are responsible for intracellular binding of UCB (Brito et al., 2006). Such binding reduces the intracellular pool of free UCB, thus contributing to protect cells and preventing the back flux of UCB. Studies of the development profile of GST proteins showed that its alpha subunit, which displays the highest affinity for UCB, is absent or down-regulated in newborn pups (Gazzin et al., 2012b), being detected only 10 days after birth in mouse choroidal cells (Beiswanger et al., 1995). Accordingly, this subunit was not detected in human fetal CP, but was detected in CP from adults (Carder et al., 1990). Therefore, in CP epithelial cells UCB-binding to GST appears to be less efficient during development than at the adult stage, which may contribute to the vulnerability of the CNS to UCB neurotoxicity in the neonatal period.

UCB is among the substrates of the ATP-binding cassette (ABC) transporters P-glycoprotein (P-gp; $\mathrm{ABCB} 1$ ) and $\mathrm{ABCC} 1$, which have been proposed to modulate the brain content of UCB (Gazzin et al., 2012a; Ghersi-Egea et al., 2009). In fact, the complementary expression of these $A B C$ transporters, with P-gp expressed at the BBB and MRP1 at the BCSFB, appear as strategic to limit CNS uptake and retention of UCB in neonates (Daood et al., 2008; Gazzin et al., 2012a). Of notice, a consistent decrease in Mrp1 expression in the $\mathrm{CP}$ of the lateral and 4th ventricles was observed in icteric Gunn rats (Gazzin et al., 2011), which may further contribute to the UCB noxious effects. In addition, or alternatively to the UCB clearance from the CSF by MRP1, is the existence of a number of transporters of the organic anion transport polypeptide (OATP) family in CP epithelial cells that may as well contribute to the clearance of UCB from the CNS (Ghersi-Egea et al., 2009), which remains unexplored. Collectively, it is conceivable that the uptake of UCB at the apical membrane of the choroidal tissue, coupled to an active efflux into the blood via the basolateral MRP1 pump acts as a mechanism accelerating the clearance of UCB from the CNS, which has implications during early post-natal development. The immaturity of such transport system in the neonatal period might impair the elimination of UCB from the brain and underlie the increased vulnerability of the neonate to UCB neurotoxicity.

\subsection{In young adults, in multiple sclerosis}

MS is a chronic progressive inflammatory disease of the CNS. In developed countries, it is the second cause of neurological disability in young adults, representing a high burden for the patient, the family and the resources of the health system (Borreani et al., 2014). MS is a complex disease in which there is a strong immune response against the myelin sheath of CNS axons; its underlying mechanisms remain however only partially understood. One of most notable aspects of the disease is the migration of peripheral inflammatory cells, through the brain barriers, towards the CNS. Specifically, the CP represents a site for lymphocyte entry in the CSF and for CSF antigens presentation. Among other characteristics, the $\mathrm{CP}$ of MS brains display T lymphocytes in vessels and stroma, vascular cell adhesion molecule-1 expression on endothelia and intense HLA-DR (human leukocyte antigen-antigen D related) immunostaining in $\mathrm{CP}$ and epiplexus cells (Vercellino et al., 2008). Additionally, the up-regulation of intercellular adhesion molecule- 1 in the $\mathrm{CP}$ epithelium and the de novo expression of mucosal addressin cell adhesion molecule- 1 in mice of the experimental autoimmune encephalomyelitis (EAE) model of MS further corroborates that the $\mathrm{CP}$ is an essential structure in the entry of cells of the immune system into the brain (Engelhardt et al., 2001). Of interest, mice lacking CCR6 ( $\mathrm{C}-\mathrm{C}$ motif chemokine receptor 6), a chemokine receptor characteristic of $\mathrm{T}(\mathrm{H})-17$ cells, develop $\mathrm{T}(\mathrm{H})-17$ responses but are highly resistant to the induction of EAE. Importantly, the CCR6 ligand CCL20 (C-C motif chemokine ligand 20) is constitutively expressed in epithelial cells of CP in mice and in humans, which suggests a role for the CP in the TH17 cell entry into the brain (Reboldi et al., 2009) (Fig. 1). This is further supported by the observation that disease susceptibility to EAE is restored by transfer of wild-type T cells to CCR6-null mice (Reboldi et al., 2009). The CP seems to be a relevant target structure in MS since $T$ cells were shown to enter the brain before disease onset and to trigger massive CCR6-independent recruitment of effector T cells across activated parenchymal vessels, which suggest that the $\mathrm{CP}$ is the initial site of $\mathrm{T}$ cell enter into the brain, before a further massive T-cell entry through the BBB (Reboldi et al., 2009). Of notice, B-cell infiltration observed in the EAE mouse model was restricted to CSF-filled extraventricular compartments (Schmitt et al., 2012). In accordance with the role of the CP in immune cells entry are the results from expression studies both in the relapse-remitting and in the chronic EAE mouse models, showing alterations in the genes encoding for adhesion molecules, chemokines and cytokines (Marques et al., 2012; Murugesan et al., 2012). Still to investigate is whether sex differences in the CP transcriptome are related with the increased susceptibility of women to MS (Voskuhl and Gold, 2012). Striking is the observation that rodent EAE models of MS are established only in particular mouse strains, and 
that females are more susceptible for the induction; as such most animal studies are done in female rodents. Interestingly, the expression of Ccr6 is higher ( 1.28 fold) while that of $C c l 20$ in lower ( 0.600 fold) in male versus female (Table 1 ). Whether the overall balance of both may contribute to the higher susceptibility of female rodent to EAE deserves further consideration.

\subsection{In aging and in Alzheimer's disease}

Life expectancy is increasing worldwide. Current challenges in public health and in health care research are, therefore, focused on increasing the number of healthy years in the aged population (Prince et al., 2013). Age is associated with cognitive and memory decline, which may precede $\mathrm{AD}$, the principal cause of dementia in the elderly (Andrieu et al., 2015; Ballard et al., 2011).

During aging, several brain structures shrink, as is the case of the prefrontal cortex and the hippocampus, regions that regulate complex functions including memory. The $\mathrm{CP}$ also presents age-related changes. Specifically, aged CP epithelial cells present a general atrophy, decreasing in height, total volume and length of the apical microvilli (Marques et al., 2013). In addition, the blood vessel walls of the CP become thicker (Marques et al., 2013). As a consequence of the overall brain shrinkage, the ventricular space expands. In addition, the overall CSF homeostasis is altered. Not only the CP synthetic capacity decreases, as revealed by the lower expression of key proteins (carbonic anhydrase II and aquaporin 1) for CSF production (Masseguin et al., 2005), and consequently CSF production diminishes, but also clearance of CSF out of the brain is delayed (May, 1990) (Fig. 1). These alterations in the CP morphology and activity are likely to impact on brain function and deterioration since the excretion of toxic compounds is compromised. In addition, down-regulation of receptors and transporters that scavenge toxic compounds is observed in the elder and in $\mathrm{AD}$.

One of the characteristic pathological features of $A D$ is the formation of amyloid plaques, mostly composed of $A \beta$ whose concentration is increased. This increase may result either from augmented production and/or decreased clearance of the $A \beta$ peptide out of the brain. In agreement, excretion of $A \beta$ has been shown to be impaired in several animal models of AD (Crossgrove et al., 2005; Zlokovic et al., 1996). Additionally, it has been suggested that the accumulation of $A \beta$ in the $C P$ further enhances the disruption of the BCSFB (Marco and Skaper, 2006; Vargas et al., 2010), which may impact on neurodegeneration. Of relevance when addressing clearance is the knowledge on the specific efflux transporter systems present at the brain barriers. The efflux of toxins and harmful substances/metabolites, mostly against a concentration gradient, is reached by $A B C$ transporters (Fig. 1). The family of $A B C$ transporter comprises 48 known human $A B C$ transporter genes and builds up one of the largest protein superfamily currently known. These can be subdivided into 7 subfamilies referred to as $A$ to $G$ (Schinkel and Jonker, 2003). Members of the B- and C-family (ABCB1, $A B C C 1$ through $A B C C 6$ ) were confirmed to be expressed at the $\mathrm{CP}$ in man (Niehof and Borlak, 2009). One of the most prominent mechanisms of $\mathrm{ABC}$ transporter activity is the multidrug resistance, which is thought to be the cause for the resistance of cancer cells to chemotherapeutic drugs (Borst et al., 2000). An association between insufficient transporter activity and the development of neurodegenerative diseases, to which $A D$ belongs, is revealed by several studies. Especially ABCB1 and $\mathrm{ABCC} 1$, by exporting metabolites from the $\mathrm{CP}$ cells, brain capillary endothelial cells and ISF/CSF into the blood, are in the focus of AD research, as numerous findings underline their importance for the transport of $A \beta$ (Pahnke et al., 2014a; Pahnke et al., 2014b; Pahnke et al., 2009; Pahnke et al., 2008).

The ABCB1 transporter plays an important role in maintaining the brain's homeostasis by transporting a wide range of different metabolites, such as cationic amphiphilic and lipophilic compounds. Its overexpression mediates resistance to a variety of hydrophobic chemotherapeutic substrates facilitating resistance to treatment of epilepsy (Loscher et al., 2011). ABCB1 has been earlier described to transport $A \beta$ (Lam et al., 2001) and its expression was found inversely correlated with $A \beta$ deposition (Vogelgesang et al., 2002). The knockout of $A B C B 1$ combined with $A D$ mouse models was found to lead to the increase in the formation of $A \beta$ plaques (Cirrito et al., 2005; Krohn et al., 2011), which was reverted by restoring ABCB1 activity (Hartz et al., 2010). In humans, ABCB1 function was assessed using in vivo $(R)-\left[{ }^{11} \mathrm{C}\right]$ verapamil as tracer for positron emission tomography investigations and it was found to be diminished at the BBB in patients with $A D$ compared to healthy elderly (van Assema et al., 2012). However, decreased $\mathrm{BBB} A B C B 1$ function was also found in aged brains, even without $\mathrm{AD}$, especially in the internal capsule, corona radiata white matter and in orbitofrontal regions as assessed by Bartels et al. (2009) and might thereby precede AD onset. Dysfunctional ABCB1 was shown to play also an important role in other neurodegenerative diseases like Parkinson's disease (Kortekaas et al., 2005).

ABCC1 can be found in high levels at the basolateral membrane of epithelial cells of the CP. It was first identified in cell lines resistant to anthracycline (Cole et al., 1994). In contrast to ABCB1, ABCC1 functions by transporting amphipathic organic anionic compounds, especially hydrophobic drugs and conjugates of glutathione, glucuronic acid or sulfate (Evers et al., 1996; Leier et al., 1994; Loe et al., 1996). Being a major landmark of the $\mathrm{CP}, \mathrm{ABCC} 1$ was reported to play an important role in the excretion of $A \beta$ from the brain. Using a mouse model for $A D$ with knocked-out $A B C C 1$, it was possible to detect a 12-14 fold increase in the brain $A \beta$ when compared to control mice. Furthermore, a pharmacological activation of the transporter using thiethylperazine led to significantly reduced soluble $A \beta$, number of $A \beta$ plaques as well as a reduction in the plaque size and was stated as a promising therapeutic option for AD treatment (Krohn et al., 2011).

A final note on the $\mathrm{CP}$ transcriptome, since recent findings in microarray studies have shown that it displays a specific ontogenic expression profile from young adulthood to old mice, particularly in genes that regulate the cellular circadian rhythm. Moreover, the $\mathrm{CP}$ of AD mouse models presented an anticipation of such ontogeny profile (Mesquita et al., 2015). J20 mice presented a high expression of type II interferon (IFN) genes in the CP at 3 months, which became lower than in WT at 5-6 and 11-12 months (Mesquita et al., 2015). Strikingly, the CP of the J20 AD mouse model displayed an overall overexpression of type I IFN response genes at different ages (3, 5-6 and 11-12 months). Furthermore, this increase in type I IFN-dependent genes has also been reported in aged human brains. Importantly, the blockage of IFN-I signaling within the aged mouse brain partially restored cognitive function and hippocampal neurogenesis and re-established IFN-II-dependent CP activity (Baruch et al., 2014), which is a promising observation in light of the possibility to modulate disease progression. Whether the same blockage in $\mathrm{AD}$ is able to restore the cognitive decline is still unexplored.

Altogether, alterations in the transporter activity and CP structure and function in aging may contribute to and/or accelerate disease mechanisms such as those observed in AD. Understanding how (in which precise conditions) and when (in time) the CP loses control of homeostasis will certainly provide novel mechanisms of intervention for diseases of the CNS.

\section{Conclusions}

This review intended to highlight particular features of the $\mathrm{CP}$ in health and in disease (Fig. 1). It is expected to convince the reader that the CP deserves several additional lines in text books of physiology and medicine. Particularly, its role in the production of CSF; in the transfer of molecules and cells into and out of the brain, which is relevant for nutrition, neurogenesis, metabolic clearance and treatment of brain diseases; in specific homeostatic mechanisms, and in the context of diseases of the newborns, young adult and the elder. A challenging issue relates with the sex-differences, which may justify the higher 
prevalence of some diseases in men or women. It is therefore important to raise awareness for including both sexes when searching for mechanisms of diseases of the CNS. Altogether, the concept of brain barriers must be revisited. Looking at the BCSFB is looking much further than to just at one of the brain barriers. It is to unravel the roles of a key structure that participates in brain homeostasis and that places the brain and the periphery in homeostatic interaction.

\section{Acknowledgements}

The work at ICVS/3B's has the support of Portuguese North Regional Operational Program (ON.2 - O Novo Norte) under the National Strategic Reference Framework (QREN), through the European Regional Development Fund (FEDER). JCS, FM and JAP have the support of a fellowship from Bial Foundation through Grant 217/12.

Fernanda Marques is a recipient of a FCT Investigator award (IF/ 00231/2013) of the Fundação para a Ciência e Tecnologia (FCT, Portugal).

The work by JP has been financed by the following grants: Deutsche Forschungsgemeinschaft DFG PA930/9, DFG PA930/12; VIAA Latvia NFI/ R/2014/023; Leibniz Society SAW-2015-IPB-2; HelseSØ No: 2016062; Norsk forskningsrådet: NFR251290, NFR246392, NFR248772, JPND NeuroGEM NFR247179, JPND PROP-AD NFR260786.

The work by CS has been supported by the Portuguese Foundation for Science and Technology (FCT, Portugal - hrrp://www.fct.pt) project grants (PTDC/SAU-NEU/114800/2009); and by FEDER funds through the POCI - COMPETE 2020 - Operational Programme Competitiveness and Internationalisation in Axis I - Strengthening research, technological development and innovation (Project No. 007491) and National Funds by FCT - Foundation for Science and Technology (Project UID/ Multi/00709).

The work by $\mathrm{AB}$ at Faculty of Pharmacy, Universidade de Lisboa, was funded, in part, by iMed.ULisboa (UID/DTP/04138/2013) from Fundação para a Ciência e a Tecnologia (FCT), Portugal.

The funding agencies had no role in the study design, collection, analysis and interpretation of data, writing of the manuscript or decision on submitting the manuscript for publication.

The authors declare no conflicts of interest.

\section{References}

Ahdab-Barmada, M., Moossy, J., 1984. The neuropathology of kernicterus in the premature neonate: diagnostic problems. J. Neuropathol. Exp. Neurol. 43, 45-56.

Alves, C.H., et al., 2009. Androgen receptor is expressed in murine choroid plexus and downregulated by 5alpha-dihydrotestosterone in male and female mice. J. Mol. Neurosci. 38, 41-49.

Andrieu, S., et al., 2015. Prevention of sporadic Alzheimer's disease: lessons learned from clinical trials and future directions. Lancet Neurol. 14, 926-944.

Ariza, J., et al., 2015. Dysregulated iron metabolism in the choroid plexus in fragile X-associated tremor/ataxia syndrome. Brain Res. 1598, 88-96.

Aspelund, A., et al., 2015. A dural lymphatic vascular system that drains brain interstitial fluid and macromolecules. J. Exp. Med. 212, 991-999.

Bakulski, K.M., et al., 2012. Alzheimer's disease and environmental exposure to lead: the epidemiologic evidence and potential role of epigenetics. Curr Alzheimer Res. 9 563-573.

Ballard, C., et al., 2011. Alzheimer's disease. Lancet 377, 1019-1031.

Banks, W.A., et al., 1996. Leptin enters the brain by a saturable system independent of insulin. Peptides 17, 305-311.

Baraldo, M., 2008. The influence of circadian rhythms on the kinetics of drugs in humans. Expert Opin. Drug Metab. Toxicol. 4, 175-192.

Bartels, A.L., et al., 2009. Blood-brain barrier P-glycoprotein function decreases in specific brain regions with aging: a possible role in progressive neurodegeneration. Neurobiol. Aging 30, 1818-1824.

Baruch, K., et al., 2014. Aging. Aging-induced type I interferon response at the choroid plexus negatively affects brain function. Science 346, 89-93.

Behl, M., et al., 2010. Lead-induced accumulation of beta-amyloid in the choroid plexus: role of low density lipoprotein receptor protein-1 and protein kinase $C$. Neurotoxicology 31, 524-532.

Beiswanger, C.M., et al., 1995. Developmental changes in the cellular distribution of glutathione and glutathione S-transferases in the murine nervous system. Neurotoxicology 16, 425-440.

Bell, J.L., et al., 2013. Insulin-like growth factor 2 mRNA-binding proteins (IGF2BPs): posttranscriptional drivers of cancer progression? Cell. Mol. Life Sci. 70, 2657-2675.
Belmadani, A., et al., 2006. Chemokines regulate the migration of neural progenitors to sites of neuroinflammation. J. Neurosci. 26, 3182-3191.

Bigos, K.L., et al., 2009. Sex differences in the pharmacokinetics and pharmacodynamics of antidepressants: an updated review. Gend. Med. 6, 522-543.

Borreani, C., et al., 2014. Unmet needs of people with severe multiple sclerosis and their careers: qualitative findings for a home-based intervention. PLoS One 9, e109679.

Borst, P., et al., 2000. A family of drug transporters: the multidrug resistance-associated proteins. J. Natl. Cancer Inst. 92, 1295-1302.

Bresciani, G., et al., 2015. Manganese superoxide dismutase and oxidative stress modulation. Adv. Clin. Chem. 68, 87-130.

Brites, D., Brito, M.A., 2012. Bilirubin toxicity. In: Stevenson, D.K., Maisels, M.J., Watchko, J.F. (Eds.), Care of the Jaundiced Neonate. McGrawHill, New York, pp. 115-143.

Brito, M. A., Silva, R.F.M., Brites, D., Cell response to hyperbilirubinemia: a journey along key molecular events. In: C. FJ, (Ed.), New Trends in Brain Research. Nova Science Publishers, Inc., New York, 2006 pp. 1-38.

Butts, C.L., Sternberg, E.M., 2008. Neuroendocrine factors alter host defense by modulating immune function. Cell. Immunol. 252, 7-15.

Carder, P.J., et al., 1990. Glutathione S-transferase in human brain. Neuropathol. Appl. Neurobiol. 16, 293-303.

Chen, L., et al., 2015. Pathways of cerebrospinal fluid outflow: a deeper understanding of resorption. Neuroradiology 57, 139-147.

Cirrito, J.R., et al., 2005. P-glycoprotein deficiency at the blood-brain barrier increases amyloid-beta deposition in an Alzheimer disease mouse model. J. Clin. Invest. 115, 3285-3290.

Cole, S.P., et al., 1994. Pharmacological characterization of multidrug resistant MRPtransfected human tumor cells. Cancer Res. 54, 5902-5910.

Combarros, O., et al., 2010. The dopamine beta-hydroxylase $-1021 \mathrm{C} / \mathrm{T}$ polymorphism is associated with the risk of Alzheimer's disease in the epistasis project. BMC Med. Genet. 11, 162.

Crandall, J.E., et al., 2011. Retinoic acid influences neuronal migration from the ganglionic eminence to the cerebral cortex. J. Neurochem. 119, 723-735

Crossgrove, J.S., et al., 2005. The choroid plexus removes beta-amyloid from brain cerebrospinal fluid. Exp. Biol. Med. (Maywood) 230, 771-776.

Cserr, H.F., 1971. Physiology of the choroid plexus. Physiol. Rev. 51, 273-311.

Damkier, H.H., et al., 2013. Cerebrospinal fluid secretion by the choroid plexus. Physiol. Rev. 93, 1847-1892.

Daood, M., et al., 2008. ABC transporter (P-gp/ABCB1, MRP1/ABCC1, BCRP/ABCG2) expression in the developing human CNS. Neuropediatrics 39, 211-218.

Dietrich, M.O., et al., 2008. Megalin mediates the transport of leptin across the blood-CSF barrier. Neurobiol. Aging 29, 902-912.

Dunn, S.E., et al., 2015. Sex-based differences in multiple sclerosis (part I): biology of disease incidence. Curr. Top. Behav. Neurosci. 26, 29-56.

Ek, C.J., et al., 2015. Transcriptomal changes and functional annotation of the developing non-human primate choroid plexus. Front. Neurosci. 9, 82

Engelhardt, B., et al., 2001. Involvement of the choroid plexus in central nervous system inflammation. Microsc. Res. Tech. 52, 112-129.

Evers, R., et al., 1996. Basolateral localization and export activity of the human multidrug resistance-associated protein in polarized pig kidney cells. J. Clin. Invest. 97, 1211-1218.

Falcao, A.M., et al., 2012a. The path from the choroid plexus to the subventricular zone: go with the flow! Front. Cell. Neurosci. 6, 34

Falcao, A.M., et al., 2012b. Topographical analysis of the subependymal zone neurogenic niche. PLoS One 7, e38647.

Faller, P., 2010. Neuronal growth-inhibitory factor (metallothionein-3): reactivity and structure of metal-thiolate clusters. FEBS J. 277, 2921-2930.

Fessner, A., et al., 2014. The transcription factor HoxB5 stimulates vascular remodelling in a cytokine-dependent manner. Cardiovasc. Res. 101, 247-255.

Fiorelli, R., et al., 2015. Adding a spatial dimension to postnatal ventricular-subventricular zone neurogenesis. Development 142, 2109-2120.

Fitsanakis, V.A., et al., 2007. Putative proteins involved in manganese transport across the blood-brain barrier. Hum. Exp. Toxicol. 26, 295-302.

Fu, X., et al., 2014. Regulation of copper transport crossing brain barrier systems by $\mathrm{Cu}-$ ATPases: effect of manganese exposure. Toxicol. Sci. 139, 432-451.

Gazzin, S., et al., 2011. Modulation of Mrp1 (ABCc1) and Pgp (ABCb1) by bilirubin at the blood-CSF and blood-brain barriers in the Gunn rat. PLoS One 6, e16165.

Gazzin, S., et al., 2012a. Transport and metabolism at blood-brain interfaces and in neural cells: relevance to bilirubin-induced encephalopathy. Front. Pharmacol. 3, 89.

Gazzin, S., et al., 2012b. Bilirubin accumulation and Cyp mRNA expression in selected brain regions of jaundiced Gunn rat pups. Pediatr. Res. 71, 653-660.

Ghersi-Egea, J.F., et al., 1994. Localization of drug-metabolizing enzyme activities to blood-brain interfaces and circumventricular organs. J. Neurochem. 62, 1089-1096.

Ghersi-Egea, J.F., et al., 2009. Blood-brain interfaces and bilirubin-induced neurological diseases. Curr. Pharm. Des. 15, 2893-2907.

Golden, P.L., et al., 1997. Human blood-brain barrier leptin receptor. Binding and endocytosis in isolated human brain microvessels. J. Clin. Invest. 99, 14-18.

Goncalves, I., et al., 2008. Transthyretin interacts with metallothionein 2. Biochemistry 47, 2244-2251

Goncalves, I., et al., 2016. 'Smelling' the cerebrospinal fluid: olfactory signaling molecules are expressed in and mediate chemosensory signaling from the choroid plexus. FEBS J. 283, 1748-1766.

Hartz, A.M., et al., 2010. Restoring blood-brain barrier P-glycoprotein reduces brain amyloid-beta in a mouse model of Alzheimer's disease. Mol. Pharmacol. 77. 715-723.

He, J., et al., 2014. Sleep restriction impairs blood-brain barrier function. J. Neurosci. 34, 14697-14706.

Hong-Goka, B.C., Chang, F.L., 2004. Estrogen receptors alpha and beta in choroid plexus epithelial cells in Alzheimer's disease. Neurosci. Lett. 360, 113-116. 
Iliff, J.J., et al., 2012. A paravascular pathway facilitates CSF flow through the brain parenchyma and the clearance of interstitial solutes, including amyloid beta. Sci. Transl. Med. 4, 147ra111.

Iliff, J.J., et al., 2013. Brain-wide pathway for waste clearance captured by contrast-enhanced MRI. J. Clin. Invest. 123, 1299-1309.

Inestrosa, N.C., Varela-Nallar, L., 2014. Wnt signaling in the nervous system and in Alzheimer's disease. J. Mol. Cell Biol. 6, 64-74.

Jiang, H., et al., 2016. Brain iron metabolism dysfunction in Parkinson's disease. Mol. Neurobiol.

Keep, R.F., Smith, D.E., 2011. Choroid plexus transport: gene deletion studies. Fluids Barriers CNS. 8, 26.

Kortekaas, R., et al., 2005. Blood-brain barrier dysfunction in parkinsonian midbrain in vivo. Ann. Neurol. 57, 176-179.

Kratzer, I., et al., 2013. Developmental changes in the transcriptome of the rat choroid plexus in relation to neuroprotection. Fluids Barriers CNS. 10, 25.

Krohn, M., et al., 2011. Cerebral amyloid-beta proteostasis is regulated by the membrane transport protein ABCC1 in mice. J. Clin. Invest. 121, 3924-3931.

Lam, F.C., et al., 2001. Beta-amyloid efflux mediated by p-glycoprotein. J. Neurochem. 76, 1121-1128.

Lehtinen, M.K., Walsh, C.A., 2011. Neurogenesis at the brain-cerebrospinal fluid interface. Annu. Rev. Cell Dev. Biol. 27, 653-679.

Lehtinen, M.K., et al., 2011. The cerebrospinal fluid provides a proliferative niche for neural progenitor cells. Neuron 69, 893-905.

Leier, I., et al., 1994. The MRP gene encodes an ATP-dependent export pump for leukotriene C4 and structurally related conjugates. J. Biol. Chem. 269, 27807-27810.

Liddelow, S.A., et al., 2013. Mechanisms that determine the internal environment of the developing brain: a transcriptomic, functional and ultrastructural approach. PLoS One 8, e65629.

Loe, D.W., et al., 1996. ATP-dependent 17 beta-estradiol 17-(beta-D-glucuronide) transport by multidrug resistance protein (MRP). Inhibition by cholestatic steroids. J. Biol. Chem. 271, 9683-9689.

Loscher, W., et al., 2011. Do ATP-binding cassette transporters cause pharmacoresistance in epilepsy? Problems and approaches in determining which antiepileptic drugs are affected. Curr. Pharm. Des. 17, 2808-2828.

Louveau, A., et al., 2015. Structural and functional features of central nervous system lymphatic vessels. Nature 523, 337-341.

Lun, M.P., et al., 2015a. Spatially heterogeneous choroid plexus transcriptomes encode positional identity and contribute to regional CSF production. J. Neurosci. 35, 4903-4916.

Lun, M.P., et al., 2015b. Development and functions of the choroid plexus-cerebrospinal fluid system. Nat. Rev. Neurosci. 16, 445-457.

Maktabi, M.A., et al., 1990. Effects of angiotensin II on blood flow to choroid plexus. Am. J. Phys. 258, H414-H418.

Marco, S., Skaper, S.D., 2006. Amyloid beta-peptide1-42 alters tight junction protein distribution and expression in brain microvessel endothelial cells. Neurosci. Lett. 401, 219-224.

Maret, W., 2009. Molecular aspects of human cellular zinc homeostasis: redox control of zinc potentials and zinc signals. Biometals 22, 149-157.

Marques, F., et al., 2009a. Altered iron metabolism is part of the choroid plexus response to peripheral inflammation. Endocrinology 150, 2822-2828.

Marques, F., et al., 2009b. Kinetic profile of the transcriptome changes induced in the choroid plexus by peripheral inflammation. J. Cereb. Blood Flow Metab. 29, 921-932.

Marques, F., et al., 2011. Transcriptome signature of the adult mouse choroid plexus. Fluids Barriers CNS. 8, 10

Marques, F., et al., 2012. Lipocalin 2 is present in the EAE brain and is modulated by natalizumab. Front. Cell. Neurosci. 6, 33.

Marques, F., et al., 2013. Blood-brain-barriers in aging and in Alzheimer's disease. Mol. Neurodegener. 8, 38 .

Martinho, A., et al., 2010. Human metallothioneins 2 and 3 differentially affect amyloidbeta binding by transthyretin. FEBS J. 277, 3427-3436.

Masseguin, C., et al., 2005. Aging affects choroidal proteins involved in CSF production in Sprague-Dawley rats. Neurobiol. Aging 26, 917-927.

Mawhinney, L.J., et al., 2013. Gender-specific differences in the central nervous system's response to anesthesia. Transl Stroke Res. 4, 462-475.

May, C., 1990. Research on nurse-patient relationships: problems of theory, problems of practice. J. Adv. Nurs. 15, 307-315.

Mendelsohn, A.R., Larrick, J.W., 2013. Sleep facilitates clearance of metabolites from the brain: glymphatic function in aging and neurodegenerative diseases. Rejuvenation Res. 16, 518-523.

Merhar, S.L., Gilbert, D.L., 2005. Clinical (video) findings and cerebrospinal fluid neurotransmitters in 2 children with severe chronic bilirubin encephalopathy, including a former preterm infant without marked hyperbilirubinemia VIDEO. Pediatrics 116, 1226-1230

Mesquita, S.D., et al., 2012. Modulation of iron metabolism in aging and in Alzheimer's disease: relevance of the choroid plexus. Front. Cell. Neurosci. 6, 25.

Mesquita, S.D., et al., 2015. The choroid plexus transcriptome reveals changes in type I and I interferon responses in a mouse model of Alzheimer's disease. Brain Behav. Immun.

Mitchell, S.E., et al., 2009. Leptin receptor gene expression and number in the brain are regulated by leptin level and nutritional status. J. Physiol. 587, 3573-3585.

Mitsui, S., et al., 2001. Antagonistic role of E4BP4 and PAR proteins in the circadian oscillatory mechanism. Genes Dev. 15, 995-1006.

Mo, J.S., et al., 2014. The hippo signaling pathway in stem cell biology and cancer. EMBO Rep. 15, 642-656.

Murray, S., et al., 2014. Hormonal and neural mechanisms of food reward, eating behaviour and obesity. Nat. Rev. Endocrinol. 10, 540-552.

Murugesan, N., et al., 2012. Active induction of experimental autoimmune encephalomyelitis by MOG35-55 peptide immunization is associated with differential responses in separate compartments of the choroid plexus. Fluids Barriers CNS. 9, 15.
Niehof, M., Borlak, J., 2009. Expression of HNF4alpha in the human and rat choroid plexus: implications for drug transport across the blood-cerebrospinal-fluid (CSF) barrier. BMC Mol. Biol. 10, 68.

Okada, Y., et al., 2004. Retinoic-acid-concentration-dependent acquisition of neural cel identity during in vitro differentiation of mouse embryonic stem cells. Dev. Biol. $275,124-142$.

Ostrow, J.D., et al., 2004. Molecular basis of bilirubin-induced neurotoxicity. Trends Mol. Med. 10, 65-70.

Pahnke, J., et al., 2008. Clinico-pathologic function of cerebral ABC transporters - implications for the pathogenesis of Alzheimer's disease. Curr. Alzheimer Res. 5, 396-405.

Pahnke, J., et al., 2009. Alzheimer's disease and blood-brain barrier function-why have anti-beta-amyloid therapies failed to prevent dementia progression? Neurosci. Biobehav. Rev. 33, 1099-1108.

Pahnke, J., et al., 2014a. Cerebral ABC transporter-common mechanisms may modulate neurodegenerative diseases and depression in elderly subjects. Arch. Med. Res. 45, 738-743.

Pahnke, J., et al., 2014b. Alzheimer's and ABC transporters-new opportunities for diagnostics and treatment. Neurobiol. Dis. 72 Pt A, 54-60.

Parada, C., et al., 2008. All-trans retinol and retinol-binding protein from embryonic cerebrospinal fluid exhibit dynamic behaviour during early central nervous system development. Neuroreport 19, 945-950.

Prince, M., et al., 2013. The global prevalence of dementia: a systematic review and metaanalysis. Alzheimers Dement. 9 (63-75), e2.

Quadros, P.S., et al., 2007. Distribution of progesterone receptor immunoreactivity in the fetal and neonatal rat forebrain. J. Comp. Neurol. 504, 42-56.

Quintela, T., et al., 2013. Analysis of the effects of sex hormone background on the rat choroid plexus transcriptome by cDNA microarrays. PLoS One 8, e60199.

Quintela, T., et al., 2016. Sex-related differences in rat choroid plexus and cerebrospinal fluid: a cDNA microarray and proteomic analysis. J. Neuroendocrinol. 28.

Reboldi, A., et al., 2009. C-C chemokine receptor 6-regulated entry of T(H)-17 cells into the CNS through the choroid plexus is required for the initiation of EAE. Nat. Immunol.

Redzic, Z.B., Segal, M.B., 2004. The structure of the choroid plexus and the physiology of the choroid plexus epithelium. Adv. Drug Deliv. Rev. 56, 1695-1716.

Rouault, T.A., et al., 2009. Brain iron homeostasis, the choroid plexus, and localization of iron transport proteins. Metab. Brain Dis. 24, 673-684.

Saunders, N.R., et al., 2015. Influx mechanisms in the embryonic and adult rat choroid plexus: a transcriptome study. Front. Neurosci. 9, 123.

Scheiermann, C., et al., 2013. Circadian control of the immune system. Nat. Rev. Immunol. $13,190-198$.

Schinkel, A.H., Jonker, J.W., 2003. Mammalian drug efflux transporters of the ATP binding cassette (ABC) family: an overview. Adv. Drug Deliv. Rev. 55, 3-29.

Schmitt, C., et al., 2012. Brain leukocyte infiltration initiated by peripheral inflammation or experimental autoimmune encephalomyelitis occurs through pathways connected to the CSF-filled compartments of the forebrain and midbrain. J. Neuroinflammation 9, 187.

Smith, P.M., Ferguson, A.V., 2014. Metabolic signaling to the central nervous system: routes across the blood brain barrier. Curr. Pharm. Des. 20, 1392-1399.

Spector, R., Johanson, C.E., 2014. The nexus of vitamin homeostasis and DNA synthesis and modification in mammalian brain. Mol. Brain. 7, 3.

Spector, R., et al., 2015. A balanced view of choroid plexus structure and function: focus on adult humans. Exp. Neurol. 267, 78-86.

Strazielle, N., Ghersi-Egea, J.F., 2015. Efflux transporters in blood-brain interfaces of the developing brain. Front. Neurosci. 9, 21.

Thouvenot, E., et al., 2006. The proteomic analysis of mouse choroid plexus secretome reveals a high protein secretion capacity of choroidal epithelial cells. Proteomics 6 5941-5952.

Tomas, J., et al., 2016. "Tasting" the cerebrospinal fluid: another function of the choroid plexus? Neuroscience 320, 160-171.

van Assema, D.M., et al., 2012. No evidence for additional blood-brain barrier P-glycoprotein dysfunction in Alzheimer's disease patients with microbleeds. J. Cereb. Blood Flow Metab. 32, 1468-1471.

Vargas, T., et al., 2010. Abeta accumulation in choroid plexus is associated with mitochondrial-induced apoptosis. Neurobiol. Aging 31, 1569-1581.

Vercellino, M., et al., 2008. Involvement of the choroid plexus in multiple sclerosis autoimmune inflammation: a neuropathological study. J. Neuroimmunol. 199, 133-141.

Vogelgesang, S., et al., 2002. Deposition of Alzheimer's beta-amyloid is inversely correlated with P-glycoprotein expression in the brains of elderly non-demented humans. Pharmacogenetics 12, 535-541.

Voskuhl, R.R., Gold, S.M., 2012. Sex-related factors in multiple sclerosis susceptibility and progression. Nat. Rev. Neurol. 8, 255-263.

Wolburg, H., Paulus, W., 2010. Choroid plexus: biology and pathology. Acta Neuropathol 119, 75-88.

Yin, R., et al., 2013. Ascorbic acid enhances Tet-mediated 5-methylcytosine oxidation and promotes DNA demethylation in mammals. J. Am. Chem. Soc. 135, 10396-10403.

Yokel, R.A., 2009. Manganese flux across the blood-brain barrier. Neruomol. Med. 11, 297-310.

Zheng, W., 2001. Toxicology of choroid plexus: special reference to metal-induced neurotoxicities. Microsc. Res. Tech. 52, 89-103.

Zheng, W., Monnot, A.D., 2012. Regulation of brain iron and copper homeostasis by brain barrier systems: implication in neurodegenerative diseases. Pharmacol. Ther. 133 177-188.

Zlokovic, B.V., et al., 1996. Glycoprotein 330/megalin: probable role in receptor-mediated transport of apolipoprotein J alone and in a complex with Alzheimer disease amyloid beta at the blood-brain and blood-cerebrospinal fluid barriers. Proc. Natl. Acad. Sci. U. S. A. $93,4229-4234$. 